\section{Usefulness of endoscopic ultrasound for the diagnosis of hemosuccus pancreaticus}

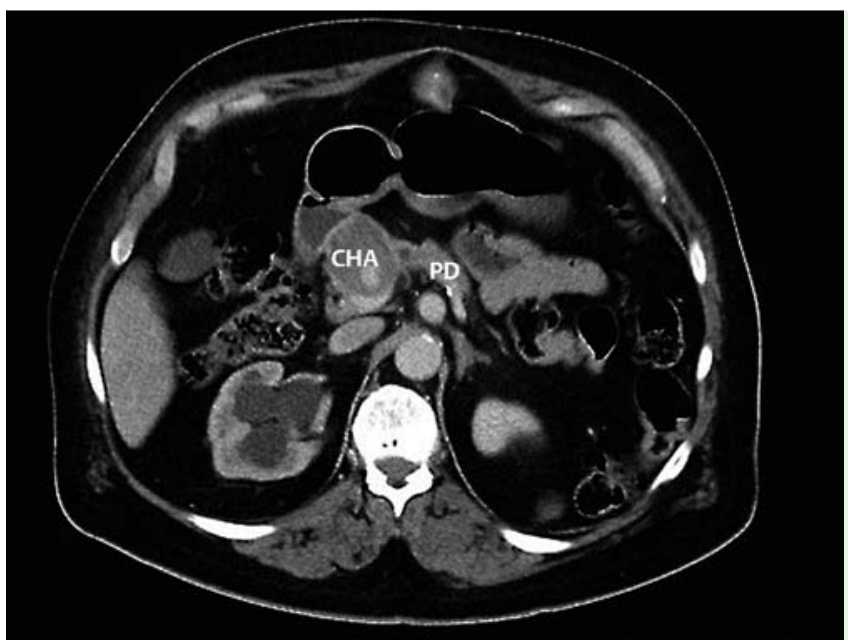

Fig. 1 Computed tomography in a 62-year-old man with severe intermittent gastrointestinal bleeding showing the aneurysm of the common hepatic artery and dilated main pancreatic duct. CHA, common hepatic artery; PD, pancreatic duct.

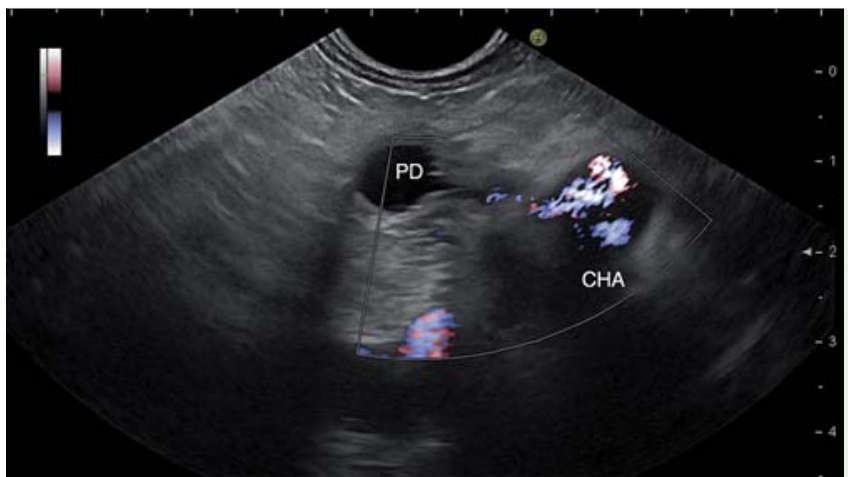

Fig. 2 Endoscopic ultrasound showing a fistula between the main pancreatic duct and common hepatic artery identified with power Doppler. CHA, common hepatic artery; PD, pancreatic duct.

Hemosuccus pancreaticus, hemorrhage from the papilla of Vater via the pancreatic duct, is a rare entity. It is the least frequent cause of upper gastrointestinal bleeding ( 1 in 5000) and is usually caused by chronic pancreatitis, pseudo-aneurysm, or pancreatic tumors [1]. Only $2 \%$ of HP involves the hepatic arteries [2]. HP is difficult to diagnose because the bleeding is intermittent and not always detectable with endoscopy. Other examinations used are computed tomography (CT), magnetic resonance imaging (MRI), selective angiography, and abdominal ultrasound. Endoscopic ultrasound (EUS) is not generally used in the diagnosis of HP. A 62-year-old man was admitted with severe intermittent gastrointestinal bleeding. He underwent gastroscopy, colonoscopy, and capsule endoscopy without signs of bleeding. The patient had no pre- vious history of pancreatitis or infection. On CT and MRI scan with angiography, an aneurism of the common hepatic artery (CHA) and dilatation of the main pancreatic duct towards the tail of the pancreas were found ( $\bullet$ Fig. 1). There was no apparent source of bleeding. EUS was then performed, and showed a partially thrombosed CHA aneurysm of $5 \mathrm{~cm}$. In addition, a fistula between the CHA and pancreatic duct could be identified with power Doppler ( Fig.2). The subsequent operation revealed a thrombosed arteriosclerotic aneurysm in the CHA with a fistula to the pancreatic duct. The aneurysm was excluded and a reversed vein graft was implanted. The postoperative course was uneventful.

To the best of our knowledge, this is the third case report of severe gastrointestinal bleeding as a result of a fistula between the CHA and pancreatic duct $[3,4]$. The use of EUS for the diagnosis of this rare condition has not been reported before, but seems feasible. Our case suggests that EUS could be advantageous when a vascular malformation is suspected.

Endoscopy_UCTN_Code_CCL_1AF_2AZ_3AD

Competing interests: None

\section{Khanh Do-Cong Pham ${ }^{1}$, Gustav Peder- sen $^{2}$, Henrik Halvorsen ${ }^{2}$, Dag Jøssang ${ }^{3}$, Roald Havre Flesland ${ }^{1,4}$, Georg Dim- cevski Gjorgji ${ }^{1,4}$}

${ }^{1}$ Department of Medicine, Haukeland University Hospital, Bergen, Norway ${ }^{2}$ Department of Vascular Surgery, Haukeland University Hospital, Bergen, Norway

${ }^{3}$ Department of Radiology, Haukeland University Hospital, Bergen, Norway

${ }^{4}$ Institute of Medicine, University of Bergen, Bergen, Norway

\section{References}

1 Rammohan A, Palaniappan R, Ramaswami S et al. Hemosuccus pancreaticus: 15-year experience from a tertiary care GI bleed centre. ISRN Radiology 2013, Article ID 191794

2 Bismuth H. Hemobilia. N Engl J Med 1973; 288: 617-619

3 Sandblom $P$. Gastrointestinal hemorrhage through the pancreatic duct. Ann Surg 1970; 171: $61-66$

4 Werner C, Bonnevie B. Gastrointestinal bleeding from a fistula between an aneurysm of the hepatic artery and the pancreatic duct. Eur J Vasc Surg 1993; 7: 95-97

\section{Bibliography}

Dol http://dx.doi.org/

10.1055/s-0034-1377641

Endoscopy 2014; 46: E528

(c) Georg Thieme Verlag KG

Stuttgart · New York

ISSN 0013-726X

\section{Corresponding author}

\section{Khanh Do-Cong Pham, MD}

Section of Gastroenterology

Department of Medicine

Haukeland University Hospital

Jonas Lies vei 65

5051 Bergen

Norway

Fax: +47-5-3414078

phamkdc@gmail.com 